\title{
Preventing, minimizing, and managing pain in patients with chronic wounds: challenges and solutions
}

This article was published in the following Dove Press journal:

Chronic Wound Care Management and Research

25 July 2016

Number of times this article has been viewed

\author{
Thomas E Serena' \\ Raphael A Yaakov' \\ Saima Aslam ${ }^{1,2}$ \\ Rummana S Aslam² \\ 'Clinical Research, SerenaGroup \\ Wound and Hyperbaric Centers, \\ Cambridge, MA, ${ }^{2}$ Center for Advanced \\ Wound Care, Hackensack University \\ Medical Center, Hackensack, NJ, USA
}

Correspondence: Thomas E Serena Clinical Research, SerenaGroup Wound and Hyperbaric Centers, 90 Sherman Street, Cambridge, MA 02140, USA

Tel +l 6179455225

Email serena@serenagroups.com
Abstract: It is widely known that pain frequently accompanies certain types of chronic wounds, most notably arterial insufficiency ulcers, venous leg ulcers, and diabetic foot ulcers; however, until recently, pain associated with chronic wounds has not received much attention. A limited number of studies have explored the impact of painful wounds on the quality of life. Nonetheless, these early works have brought wound-related pain (WRP) to the forefront and highlighted the importance of pain assessment and management in enhancing the quality of life and improving wound healing outcomes. This review of the current literature on WRP presents an overview of pain pathophysiology, clinical features of WRP, and the limitations of pain assessment methods, and offers clinical pearls on preventing, managing, and minimizing WRP.

Keywords: chronic wounds, pain assessment, pain management, patient experience, quality of life

\section{Introduction}

In the recent years, there has been an increased awareness of the importance of pain assessment and management in patients with chronic wounds. However, only a limited number of studies have explored the impact of wound-related pain (WRP) on wound healing and quality of life (QOL). While the prevalence of pain in chronic wounds is not well documented, it is largely known that unremitting and debilitating pain accompanies different types of chronic wounds, including arterial insufficiency ulcers (AIU), venous leg ulcers (VLU), and diabetic foot ulcers (DFU). An evidence-based approach to pain management improves patient satisfaction and wound healing outcomes for several reasons, including better adherence to the prescribed treatment regimen.

Pain measurement and management represent a complex and difficult problem. First of all, the perception of pain cannot be measured directly. It depends largely on the individual experiencing it (Figure 1). Many patients do not openly discuss their pain. There are wide cultural differences in the expression of pain. Furthermore, it is important to note that men and women react to pain differently. ${ }^{1}$ Differences in personal and cultural backgrounds can contribute to large variations in experience and expression of pain, which may translate to potential errors in pain assessment and management. ${ }^{2}$ Thus, to address the complexity of pain management, an integrated model of care that is culturally sensitive is needed to optimize patient satisfaction and outcomes.

\section{Pathophysiology of pain}

In brief, pain can be either nociceptive, caused by damage to the body tissue, or neuropathic, arising from dysfunction or injury of the sensory axons of the peripheral or central nerves. 


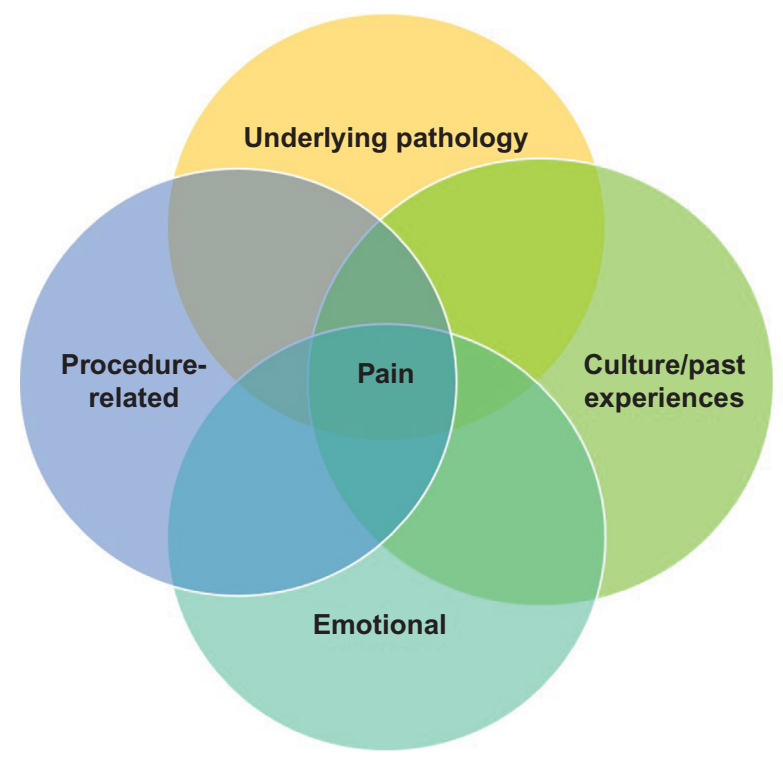

Figure I Pain in chronic wounds: a multifaceted problem.

Nociceptors respond to several forms of stimulation, including thermal, chemical, and mechanical. Injured and inflamed tissues release a wide range of chemical mediators, including prostaglandins, leukotrienes, histamine, bradykinin, acetylcholine, and serotonin. These chemical mediators stimulate nociceptors or sensitize them to the effect of nociceptive stimuli. Nociceptive stimulation that activates $\mathrm{C}$ fibers can cause neurogenic inflammation that produces vasodilation and increased release of chemical mediators. ${ }^{3}$ This in turn increases inflammation, which further heightens sensitivity to pain. Substance P, also released in response to nociceptive stimulation, can contribute to development and maintenance of chronic pain. ${ }^{4,5}$

The most common cause of neuropathic pain is peripheral nerve disease. Neuropathic pain can arise through multiple and complex pathophysiological mechanisms. Increase in voltage-gated sodium channel expression can allow for decreased activation threshold and increased membrane excitability. ${ }^{6}$ Release of neuropeptides has also shown to result in neuronal hyperexcitability, which can contribute to persistent pain via activation of A- $\beta$ and A- $\delta$ afferent fibers. Moreover, mediators, such as chemokines, substance $\mathrm{P}$, and TNF $\alpha$, can also enhance neuroexcitability.

In summary, peripheral mechanisms, peripheral-central mechanisms, and central mechanisms play a role in chronic pain. ${ }^{7}$ Peripheral mechanisms involve persistent stimulation of nociceptors. Inflammatory mediators increase the sensitivity of the $\mathrm{C}$ fibers and lead to increased duration of pain. Peripheral-central mechanisms result from abnormal function of the peripheral and central portions of the somatosensory system. ${ }^{8}$ Central mechanisms are related to disease or injury of the central nervous system. ${ }^{9}$

\section{Pain in chronic wounds}

Pain, as Melzack ${ }^{10}$ recently proposed, is a multidimensional experience that is produced by sensory factors; existing pattern of synaptic connections, which are genetically determined; and other sources of input from the body, including attention, expectation, culture, and personality. Melzack's neuromatrix theory recognizes the range of influences that shape an individual's perception of pain and provides a broader framework for assessment, management, and treatment of WRP.

It is well known that stressors such as pain can raise cortisol levels, increase heart rate, and elevate blood pressure, which can have a negative impact on wound healing. Raised levels of cortisol can also lead to myopathy, fatigue, weakness, and a decrease in immune function. ${ }^{11}$ In a study of patients with acute surgical wounds, Broadbent et al ${ }^{12}$ found that a high level of preoperative stress was associated with reduced levels of proinflammatory cytokines, which are essential in clearing debris and infection. In a sample of patients undergoing gastric bypass surgery, McGuire et a ${ }^{13}$ revealed that persistent postsurgery pain was an important predictor of healing time. Patients who rated their pain as $<8 / 10$ on the first 2 days post-surgery experienced faster healing compared with those who reported more intense pain post-surgery. Another study by Cole-King and Harding ${ }^{14}$ also demonstrated that delayed healing was associated with higher anxiety and depression score. These findings suggest that accurate assessment and management of WRP and stress may improve wound healing.

Studies suggest that pain is often exacerbated by local treatments, such as dressing changes as well as the condition of the periwound skin. ${ }^{2,15}$ An international survey by Price et al was one of the first to focus on the patient's perspective on dressing-related pain in chronic wounds. The survey suggested that $>30 \%$ of individuals with wounds experience dressing-related pain most or all of the time and $60 \%$ of the sample reported that the pain took longer than 1 hour to resolve. ${ }^{2}$ The study further concluded that wound type is a strong indicator of WRP experience across all cultural groups; VLU and AIU were reported to be the most painful. ${ }^{2}$

Another study, a randomized crossover trial investigating pain at dressing change, confirmed that interventions such as dressing removal, debridement, and inappropriate dressing selection contribute to WRP. ${ }^{15}$ Moreover, patients with wound margin maceration and skin damage were prone to experience increased pain even before dressing changes. ${ }^{15}$ Additionally, wound care practitioners must recognize that wound cleansing may exacerbate pain. It is recommended that practitioners use warm isotonic solution during wound 
irrigation and protect the periwound skin from excoriation with an appropriate barrier after the irrigation. ${ }^{16,17}$

Another common cause of pain in chronic wounds is tissue damage, which is referred to as nociceptive pain. ${ }^{18}$ Nociceptive pain can be acute or chronic and is generally localized to the wound and the surrounding tissues. This is often described as a sharp or stabbing pain and can be persistent due to continual stimulation of nociceptors in the area of tissue damage. Topical anesthetics, local anesthetics, or antianxiety medications may be used before debridement. The use of nontraumatic dressing, soaking a dressing before removal, and allowing patient control can minimize this type of pain. ${ }^{19}$

Neuropathic pain, commonly described as burning, tingling, or shooting sensation, is associated with diabetic foot ulcers (DFU). Nerve damage is a long-term complication of diabetes. Diabetic neuropathic pain often appears during periods of diminished external sensory stimulation. ${ }^{20}$ Since pain is often worse at night, it can seriously disrupt sleep and can greatly affect QOL. Neuropathic pain can be difficult to control. For patients with painful diabetic neuropathy, duloxetine, amitriptyline, or pregabalin is recommended. A management of blood glucose can reduce the progression of nerve damage.

Continuous burning pain, which typically aggravates after reclining or elevating, is the distinguishing characteristic of rest pain. Patients with critical limb ischemia (CLI) have severely reduced microcirculation. Inadequate perfusion can lead to endothelial dysfunction and inflammation. Patients with CLI require immediate treatment to reestablish blood flow. Thus, the earlier a diagnosis is made, the earlier a treatment can begin to reduce pain and improve blood flow to save the leg. Adding gabapentin as an adjuvant therapy can lead to pain reduction in patients with CLI. ${ }^{21}$

Infection in chronic wounds contributes to WRP. Woundrelated bacteria can release metalloproteases and other mediators of inflammation. ${ }^{22}$ These excess inflammatory mediators lead to tissue damage and direct stimulation of peripheral pain receptors. The increase in sensitivity of the pain receptors results in an increase in the overall perception of pain. ${ }^{23,24}$ Wound practitioners should watch for the presence of surrounding skin pain, warmth, and swelling as these signs indicate the possibility of soft tissue cellulitis. ${ }^{22}$
In making wound infection assessments, practitioners should use the mnemonic NERDS (Nonhealing wounds, Exudative wounds, Red and bleeding wound surface granulation tissue, Debris on the wound surface, Smell or unpleasant odor) for superficial infection and STONES (Size is bigger, Temperature is increased, Os probe to or exposed bone, New or satellite areas of breakdown, Exudate, erythema, edema, Smell) for deep infection. ${ }^{22}$ Timely assessment and management of bacterial burden and deep tissue infection can help reduce pain related to infection.

\section{Pain assessment}

A number of methods are available to assess pain and stress; however, most of these assessments depend on self-reporting of patients. Given the multifaceted and subjective nature of pain, the pain scales fall short on objectively quantifying the experience of pain and validating the efficacy of an intervention.

The Numeric Rating Scale (NRS), which has been widely implemented in primary care, has modest accuracy in identifying patients with clinically important pain. One of the major disadvantages of NRS is that it defines pain only in terms of its intensity (Figure 2). Moreover, when taking measurements over time, a patient's previously reported ratings may influence how he or she rates subsequent pain.

Similarly, the Visual Analog Scale (VAS) measures pain intensity by having the patient mark a pain level on a line ranging from no pain to worst possible pain. The VAS reduces bias because patients are less likely to recall the location of a previously marked line on the scale (Figure 3). Some evidence suggests that older patients can have difficulty in understanding and using the VAS.

The Verbal Pain Rating Scale (VPRS) also measures pain intensity. However, the scale may artificially categorize pain by forcing the patient to select from a list of adjectives describing pain-related symptoms. The drawback of VPRS is that it may not reflect the patient's true sensation of pain.

The Wong-Baker FACES Pain Rating Scale provides a quick and fast assessment of the emotional aspect of pain. It is an ordinal scale and consists of a limited number of categorical responses in a specific pattern. Additionally, bias is introduced as some individuals are reluctant to pick the

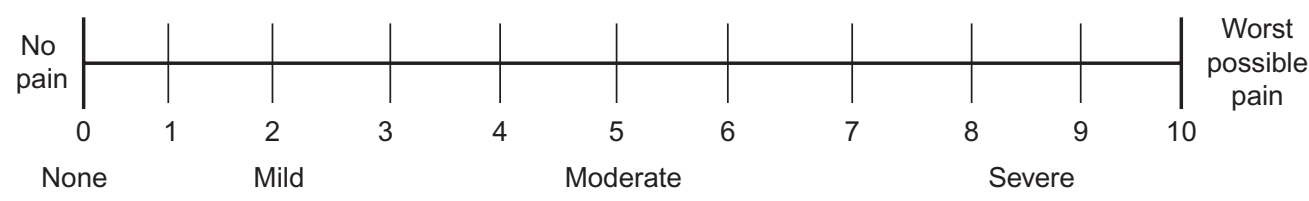

Figure 2 Numeric Pain Rating Scale. 


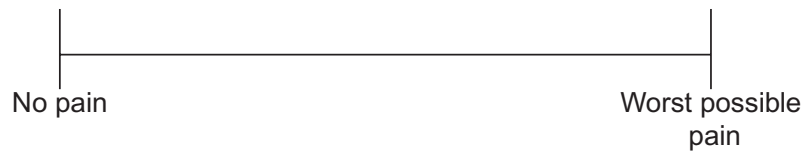

Figure 3 Visual Analog Scale.

face with tears (Figure 4). Moreover, the Wong-Baker FACES Pain Rating Scale prompts patients to report acute pain rather than chronic pain, thus limiting the range of clinical contexts in which the scale may be used.

McGill Pain Questionnaire, developed by Ronald Melzack, provides a multidimensional assessment, which goes beyond assessing pain intensity. It evaluates several different components of pain: how pain changes over time and the factors that increase or relieve it. In addition, it provides a more sensitive tool to determine treatment-related changes. In a pilot study, Roth et $\mathrm{a}^{25}$ found McGill Pain Questionnaire to be more sensitive to the pain experience than a single rating of pain intensity and that it was positively correlated with wound stage, affective stress, and symptoms of depression.

\section{Pain management}

Pharmacological therapy continues to be the mainstay in the treatment of WRP. However, as in all chronic conditions, there is the risk of dependency and the potential for abuse. The World Health Organization proposes treatment of mild-tomoderate nociceptive pain with nonopioid medications, such as acetaminophen, and nonsteroidal anti-inflammatory medications. ${ }^{26}$ Opioid analgesics should be considered for more severe pain. The use of opioids for the treatment of noncancer pain remains controversial. There continues to be concerns regarding misuse and abuse of opioids. Thus, clinicians need to actively identify and assess at-risk patients to minimize abuse and to treat pain effectively. For neuropathic pain, nonanalgesic drugs, such as tricyclic antidepressants and anticonvulsants, are recommended. Amitriptyline and gabapentin are typically used in clinical practice. Entonox, a self-administered analgesic, has also been popular; however,

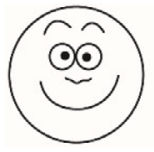

0

No

hurt

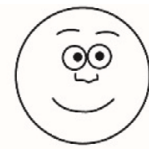

2

Hurts

little bit

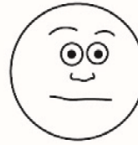

4

Hurts

little more

it is only recommended for short periods of time, such as dressing changes and procedures. ${ }^{27}$

Nonpharmacological approaches, such as diversional therapy, breathing and relaxation exercises, and music may also help eliminate some anxiety, particularly during dressing changes. It is important to note that anxiety generates an autonomic response such as muscle tension and increased heart rate, which along with past experiences may cause patients to perceive greater pain. Thus, care should be provided in a warm and calm environment that will allow the patient to relax. Finally, new techniques for pain control in the wound clinic setting need to be investigated.

\section{Discussion}

While pain is subjective, influenced by culture, emotions, and personal experiences, the molecular events that cause the nociceptor response are similar and occur uniformly. Firing of afferent fibers causes axonal release of vesicles containing neuropeptides such as substance $\mathrm{P}$, which can sensitize the nociceptor and increase its rate of firing. Cellular damage and inflammation increase concentrations of other mediators, including histamine, bradykinin, and prostaglandins. A short span of pain mediators can assist in fibroblast collagen production, cellular migration, and reepithelialization..$^{28-30}$ However, in chronic nonhealing wounds, a sustained release of pain mediators can create an imbalance that favors extended inflammation, excessive matrix metalloproteinase release, and extensive breakdown of extracellular matrix. ${ }^{28-34}$ This can delay healing or complicate the underlying disease process. The complex changing relationship between inflammatory process and wound healing needs to be addressed.

Additionally, many wound-management procedures can be painful. For example, debridement using a sharp surgical instrument can cause a considerable amount of pain. ${ }^{35}$ Trauma at dressing removal has been documented to cause pain. ${ }^{36}$ Clinicians should choose an atraumatic dressing that reduces discomfort with dressing changes and wear. It is for this reason that saline wet-to-dry dressings

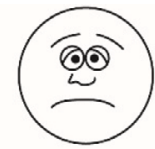

6

Hurts

even more

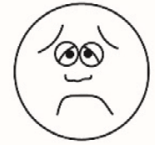

8

Hurts

whole lot

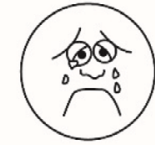

10

Hurts

worst

Figure 4 Wong-Baker FACES ${ }^{\circledR}$ Pain Rating Scale.

Notes: Reproduced from WongBaker FACES Foundation (2015). http://www. WongBakerFACES.org. ${ }^{43}$ 
are no longer recommended. Traditional dressings can be constrictive and uncomfortable causing damage to the tissue and exacerbating WRP. ${ }^{27,37}$ Modern dressings with adhesive borders can result in extensive skin tears. ${ }^{38,39} \mathrm{~A}$ dressing that does not adhere to the wound or wound margin, or causes trauma on removal, such as soft silicone products, hydrogels, alginates, and hydrofibers, can help minimize WRP. It is important that practitioners correctly match the parameters of a dressing to the key characteristics of the wound and surrounding tissues to manage wound pain effectively. ${ }^{40}$ In addition to choosing a pain-free dressing, the clinician should consider prescribing dressings that require less frequent dressing changes. It is advisable to establish dressing selection protocols for different patient needs. In patients with persistent WRP, the wound should be assessed and dressings that minimize trauma on dressing change, protect the periwound skin, and manage localized wound infection should be considered.

Patient satisfaction will soon be tied to reimbursement in the US as part of the Affordable Care Act. ${ }^{41}$ In addition, an area rapidly gaining importance for the wound practitioner is the impact of pain on wound healing. Clinicians often overlook the need for a thorough investigation of a patient's pain: Does the patient have WRP and pain with dressing changes and procedures? The patient's complaints are often dismissed due to their subjective and inconsistent nature. It is important to establish the underlying cause of WRP in the management of chronic wounds. ${ }^{42}$ The assessment of WRP should include a thorough evaluation of pain origin, such as bacterial colonization, arterial insufficiency, excessive edema, failure to achieve appropriate moisture management, and peripheral neuropathy. Once identified, the practitioner can specifically address the underlying causes contributing to the painful symptoms.

The effectiveness of pain management relies on the implementation of a culturally sensitive model of care that is patient centered, geared to the individual's specific needs, and assesses potential underlying causes of pain. Implementation of a validated pain scale along with a QOL questionnaire is an effective way to evaluate the impact of WRP on a patient's life. To effectively reduce pain and improve wound healing outcomes, practitioners also need to assess behavioral signs of stress and anxiety. By listening carefully to patients and empowering them to take an active part in their care, practitioners can improve patient experience and help reduce anxiety and stress.

\section{Disclosure}

The authors report no conflicts of interest in this work.

\section{References}

1. Bradbury J. Why do men and women feel and react to pain differently? Research suggests men and women may not process pain signals the same way. Lancet. 2003;361(9374):2052-2053.

2. Price PE, Fagervik-Morton H, Mudge EJ, et al. Dressing-related pain in patients with chronic wounds: an international patient perspective. Int Wound J. 2008;5(2):159-171.

3. Julius D, Basbaum AI. Molecular mechanisms of nociception. Nature. 2001;413(6852):203-210.

4. Dougherty PM, Willis WD. Enhanced responses of spinothalamic tract neurons to excitatory amino acids accompany capsaicin-induced sensitization in the monkey. J Neurosci. 1992;12(3):883-894.

5. Claliw DJ. The pathogenesis of chronic pain and fatigue syndromes, with special reference to fibromyalgia. Med Hypotheses. 1995;44(5): 369-378.

6. Costigan M, Scholz J, Woolf CJ. Neruropathic pain: a maladaptive response of the nervous system to damage. Annu Rev Neurosci. 2009;32:1-32.

7. Jacobson L, Mariano AJ. General considerations in chronic pain. In: Loeser JD, editor. Bonica's Management of Pain. 3rd ed. Philadelphia: Lippincott Williams \& Wilkins; 2001:241-245.

8. Hainline B. Chronic pain; physiologic, diagnostic and management considerations. Psychiatr Clin N Am. 2005;28(3):713-735.

9. Canavero S, Bonicalzi V. Central Pain Syndrome: Pathophysiology, Diagnosis and Management. New York: Cambridge University Press; 2007.

10. Melzack R. Evolution of the matrix theory of pain. The Prithvi Raz lecture: presented at the third World Conference Institute of Pain, Barcelona 2004. Pain Pract. 2005;5(2):85-94.

11. Sivamani RK, Pullar CE, Manabat-Hidalgo CG, et al. Stress-mediated increases in systemic and local epinephrine impair skin wound healing: potential new indication for beta blockers. PLoS Med. 2009;6(1):e12.

12. Broadbent E, Petrie KJ, Alley PG, Booth RJ. Psychological stress impairs wound repair following surgery. Psychosom Med. 2003;65(5):865-869.

13. McGuire L, Heffner K, Glaser R, et al. Pain and wound healing in surgical patients. Ann Behav Med. 2006;31(2):165-172.

14. Cole-King A, Harding KG. Psychological factors and delayed healing in chronic wounds. Psychosom Med. 2001;63(2):216-220.

15. Woo KY, Coutts PM, Price P, Harding K, Sibbald RG. A randomized crossover investigation of pain at dressing change comparing 2 foam dressings. Adv Skin Wound Care. 2009;22(7):304-310.

16. Bishop SM, Walker M, Rogers AA, Chen WY. Importance of moisture balance at the wound-dressing interface. $J$ Wound Care. 2003;12(4): 125-128.

17. Cuncliffe PJ, Fawcett TN. Wound cleansing: the evidence for the techniques and solutions used. Prof Nurse. 2002;12(2):95-99.

18. Clay CS, Chen WYJ. Wound pain: the need for a more understanding approach. J Wound Care. 2005;14(4):181-184.

19. Dickenson AH, Chapman V, Green GM. The pharmacology of excitatory and inhibitory amino acid-mediated events in the transmission and modulation of pain in the spinal cord. Gen Pharmacol. 1997;28(5): 633-638.

20. Sibbald GR, Armstrong DG, Orstead HL. Pain in diabetic foot ulcers. Ostomy Wound Manage. 2003;49(4 suppl):24-29.

21. Morris-stiff G, Lewis MH. Gabapentin (Neurontin) improves pain scores of patients with critical limb ischaemia: an observational study. Int $J$ Surg. 2010;8(3):212-215.

22. Sibbald GR, Woo K, Ayello E. Increased bacterial burden and infection: NERDS and STONES. Wounds UK. 2007;3(2):25-46.

23. European Wound Management Association (EWMA). Position Document: Pain at Wound Dressing Changes. London: MEP Ltd; 2002.

24. European Wound Management Association (EWMA). Position Document. Management of Wound Infection. London: MEP Ltd; 2006.

25. Roth RS, Owery JC, Hamill JB. Assessing persistent pain and its relation to affective distress, depressive symptoms, and pain catastrophizing in patients with chronic wounds: a pilot study. Am J Phys Med Rehabil. 2004;83(11):827-834. 
26. World Health Organization [webpage on the Internet]. WHO's Pain Ladder. Available from: http://www.who.int/cancer/palliative/painladder/en/. Accessed December 15, 2015.

27. Briggs M, Torra I, Bou JE. Pain at wound dressing changes: a guide to management. European Wound Management Association. Position Document: Pain at Wound Dressing Changes. London: Medical Education Partnership; 2002.

28. Schultz GS, Wysocki A. Interactions between extracellular matrix and growth factors in wound healing. Wound Repair Regen. 2009;17(2): $153-162$.

29. Chen WY, Rogers AA. Recent insights into the causes of chronic leg ulceration in venous diseases and implications on other types of chronic wounds. Wound Repair Regen. 2007;15(4):43-49.

30. Kawamoto K, Matsuda H. Nerve growth factor and wound healing. Prog Brain Res. 2004;146:369-384.

31. Summer GJ, Dina OA, Levine JD. Enhanced inflammatory hyperalgesia after recovery from burn injury. Burns. 2007;33(8):1021-1026.

32. Ye FL, Shen HD, Lin MW, Chang CY, Tai HY, Huang MH. Kelioidderived fibroblasts have a diminished capacity to produce prostaglandin E2. Burns. 2006;32(3):299-304.

33. Dray A. Inflammatory mediators of pain. Br JAnaesth. 1995;75(2):125-131.

34. Ma X, Ottino P, Bazan HEP, Bazan NG. Platelet-activating factor (PAF) induces comeal neovascularization and upregulates VEGF expression in endothelial cells. Invest Ophthalmol Vis Sci. 2004;45(9):2915-2921.
35. Sibbald RG, Goodman L, Woo KY, et al. Special considerations in wound bed preparation 2011: an update(C). Adv Skin Wound Care. 2011;24(9):415-436.

36. Woo K, Sibbald G, Fogh K, et al. Assessment and management of persistent (chronic) and total wound pain. Int Wound J. 2008;5(2): 205-215.

37. Bethell E. Why gauze dressings should not be the first choice to manage most acute surgical cavity wounds. J Wound Care. 2003;12(6): 237-239.

38. King B. Pain at first dressing change after toe nail avulsion: the experience of nurses, patients and an observer. J Wound Care. 2003;12(1):5-10.

39. King B. A review of research investigation pain and wound care. $J$ Wound Care. 2003;12(6):219-223.

40. World Union of Wound Healing Societies. Principles of Best Practice: Minimising Pain at Wound Dressing-Related Procedures. A Consensus Document. London: MEP Ltd; 2004.

41. Patient Protection and Affordable Care Act, 42 U.S.C. $\S 18001$ et seq. 2010.

42. Sibbald GR, Katchky A, Queen D. Medical management of chronic wound pain. In: White R, Harding K, editors. Trauma and Pain in Wound Care. Aberdeen: Wounds UK; 2006:134-160.

43. WongBaker FACES Foundation (2015). WongBaker FACES $®$ Pain Rating Scale. Available from: http://www.WongBakerFACES.org. Accessed June 28, 2016
Chronic Wound Care Management and Research

\section{Publish your work in this journal}

Chronic Wound Care Management and Research is an international, peer reviewed, open access, online journal publishing original research, reviews, editorials, and commentaries on the causes and management of chronic wounds and the major issues related to chronic wound management. Topics also include chronic wounds as comorbidities to other

Submit your manuscript here: https://www.dovepress.com/chronic-wound-care-management-and-research-journal

conditions, patient adherence to therapy, and the economic burden of chronic wounds. The manuscript management system is completely online and includes a very quick and fair peer review system, which is all easy to use. Visit http://www.dovepress.com/testimonials.php to read real quotes from published authors. 